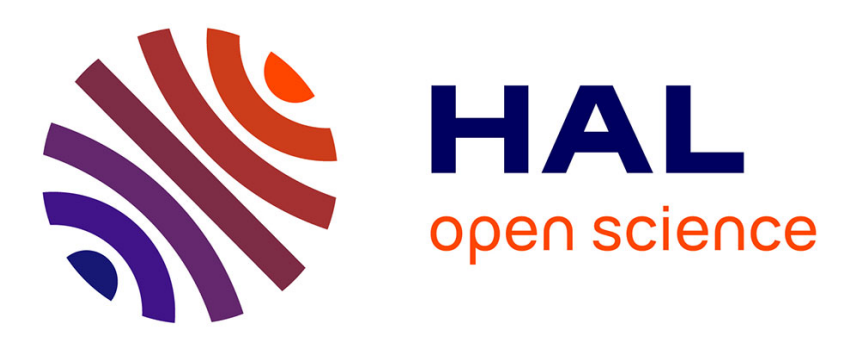

\title{
Dispositifs pour l'enregistrement graphique du quotient de deux tensions continues et variables
}

Michel Krauzman, Raymond Kara

\section{To cite this version:}

Michel Krauzman, Raymond Kara. Dispositifs pour l'enregistrement graphique du quotient de deux tensions continues et variables. Revue de Physique Appliquée, 1966, 1 (2), pp.143-144. 10.1051/rphysap:0196600102014300 . jpa-00242699

\section{HAL Id: jpa-00242699 https://hal.science/jpa-00242699}

Submitted on 1 Jan 1966

HAL is a multi-disciplinary open access archive for the deposit and dissemination of scientific research documents, whether they are published or not. The documents may come from teaching and research institutions in France or abroad, or from public or private research centers.
L'archive ouverte pluridisciplinaire HAL, est destinée au dépôt et à la diffusion de documents scientifiques de niveau recherche, publiés ou non, émanant des établissements d'enseignement et de recherche français ou étrangers, des laboratoires publics ou privés. 


\title{
DISPOSITIFS POUR L'ENREGISTREMENT GRAPHIQUE DU QUOTIENT DE DEUX TENSIONS CONTINUES ET VARIABLES
}

\author{
Par Michel KRAUZMAN et Raymond KARA, \\ Laboratoire des Recherches Physiques de la Sorbonne.
}

\begin{abstract}
Résumé. - Analyse d'un procédé classique dans lequel on remplace la tension de référence d'un potentiomètre enregistreur par la tension "Dénominateur ». Le calcul montre que le fonctionnement est inacceptable lorsque cette dernière varie dans de grandes proportions et on décrit alors un montage dans lequel cet inconvénient est supprimé. Une réalisation pratique s'est montrée satisfaisante.
\end{abstract}

Abstract. - Analysis of a conventional procedure, in which the reference e. $m$. f. of a recording potentiometer is replaced by a "Denominator" e. $\mathrm{m}$. $\mathrm{f}$. It is shown that the use is inconvenient when this e. m.f. varies over a wide range. This drawback can be avoided by a new design which is described and has given good practical results.

1. Dispositif à un potentiomètre. - 1.0. Le procédé le plus simple consiste à employer un voltmètre ou un millivoltmètre potentiométrique enregistreur d'un type commercial classique dans lequel on substitue la tension "dénominateur " $V_{\mathrm{D}}$ à la tension stabilisée de référence d'origine $V_{\mathrm{b}}$. Un tel montage est schématisé ( fig. 1).

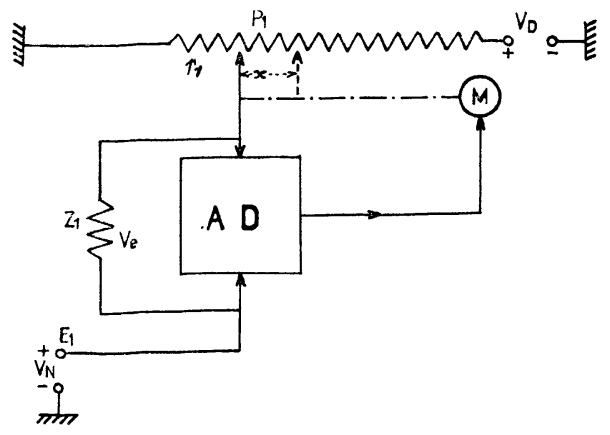

FIG. 1.

Nous appellerons : $V_{\mathbb{N}}$, la tension d'entrée $; V_{\mathrm{D}}$, la tension de référence (pile étalon, ou tension stabiisée, ou tension de comparaison); $Z_{1}$, la résistance d'entrée de l'amplificateur différentiel de gain $G$ aux bornes de laquelle apparaît une d. d. p. $V_{\mathrm{e}}$ lorsque le curseur n'est pas à l'équilibre; $P_{1}$, la résistance totale du potentiomètre de mesure ; $\rho$ sa résistivité linéaire ; $p_{1}$, la valeur de la fraction de $P_{1}$ utilisée à l'équilibre et telle que :

$$
\frac{p_{1}}{P_{1}}=\frac{V_{\mathrm{N}}}{V_{\mathrm{D}}}
$$

(on a alors $V_{\mathrm{e}}=0$ ).

1.1. Étude du fonctionnement de ce dispositif. -
Le mouvement de la plume, mécaniquement liée au curseur de $P_{1}$ est régi par l'équation :

$$
A \frac{\mathrm{d}^{2} x}{\mathrm{~d} t^{2}}+B \frac{\mathrm{d} x}{\mathrm{~d} t}+C x=0
$$

dans laquelle : $x=$ écart (supposé petit) de la position actuelle du curseur par rapport à celle d'équilibre ; $A=$ inertie mécanique (constante); $B=$ amortissement visqueux (mécanique, électrique, électronique...) (constant); $C=$ force de rappel par unité de longueur.

L'approximation des petits déplacements permet de calculer $C$ :

$C=k_{1} G V_{\mathrm{e}}=V_{\mathrm{D}} k_{1} \rho G Z_{1} /\left(P_{1} Z_{1}+P_{1} p_{1}-p_{1}^{2}\right)$.

Le gain $G$ des appareils commerciaux est réglable pour que $C$ soit égal à l'amortissement critique $C_{0}$, défini par: $B^{2}-4 A C_{0}=0$.

L'expérience montre que le fonctionnement est encore acceptable si :

$$
C_{0} / 2<C<2 C_{0} \text {. }
$$

La relation (3) indique que, pour un appareil donné, $C$ est fonction de $p_{1}$ et de $V_{\mathrm{D}}$.

La variation en fonction de $p_{1}$, pour $V_{\mathrm{D}}$ constant, est représentée (fig. 2).

La relation (4) exige que $C>C_{0} / 2$; il en sera ainsi tant que $Z_{1}>P_{1} / 4$.

Le fonctionnement d'un enregistreur n'est convenable lorsque $p_{1}$ c'est-à-dire $V_{\mathrm{N}}$ varie, que si $Z_{1}>P_{1} / 4$.

La relation (3) montre que, pour respecter (4), $V_{\mathrm{D}}$ ne doit pas varier de plus de 1 à 4 .

Le principal inconvénient de ce dispositif est donc dû au fait que le mouvement et le temps de réponse de la plume sont liés à la valeur de $V_{\mathrm{D}}$.

2. Dispositif à deux potentiomètres. - 2.0. Nous proposons ci-dessous un montage qui permet l'enre. 


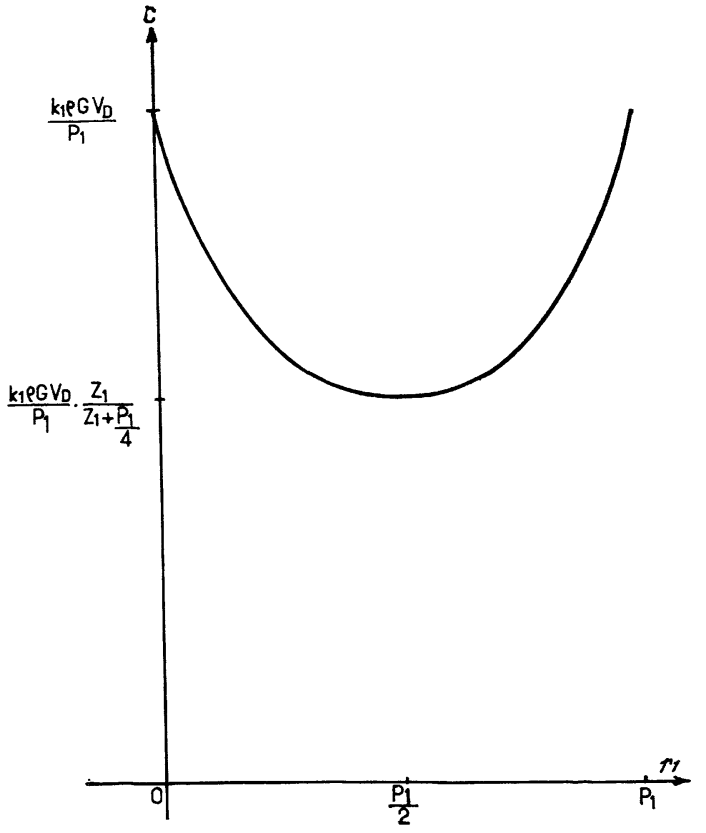

FIG. 2.

gistrement de $V_{\mathrm{N}} / V_{\mathrm{D}}, V_{\mathrm{D}}$ pouvant varier dans de très grandes proportions, par exemple de 1 à 100 et qui, de plus, permet l'enregistrement de $V_{\mathrm{D}}$. Ce montage est schématisé (fig. 3).

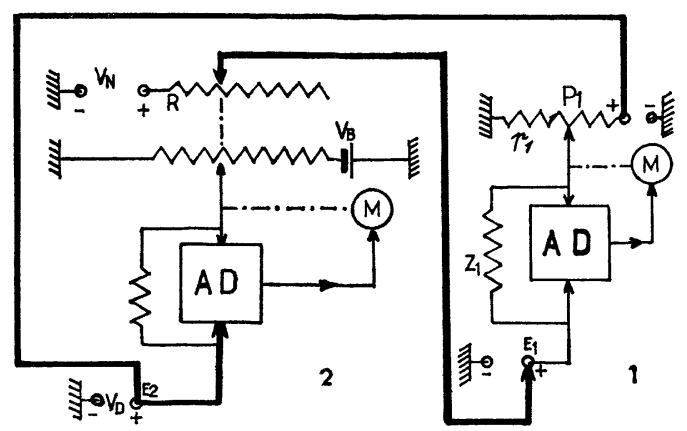

FIg. 3. - M : moteur d'asservissement de l'aiguillecurseur ; AD : amplificateur différentiel $; \mathrm{E}_{1}$ : borne d'entrée de l'enregisteur $n^{0} 1 ; E_{t_{2}}$ : borne d'entrée de l'enregistreur $n^{\circ} 2$.

2.1. On utilise un second appareil potentiométrique enregistreur, auquel on ajoute un rhéostat dont le curseur est mécaniquement lié à la plume de l'enregistreur.

La tension $V_{\mathbb{N}}$ est appliquée au premier enregistreur avec, en série, une fraction $R$ du rhéostat, proportionnelle à $V_{\mathrm{D}}: V_{\mathrm{D}}=k_{2} R$. Il en découle que la nouvelle " tension d'erreur " $V_{\mathrm{e}}^{\prime}$ s'exprime ainsi

et $C$ devient

$$
V_{\mathrm{e}}^{\prime}=Z_{1} V_{\mathrm{e}} /\left(Z_{1}+R\right)
$$

$C_{1}=k_{1} G V_{\mathrm{D}} Z_{1} \rho /\left[P_{1}\left(Z_{1}+R\right)+P_{1} p_{1}-p_{1}^{2}\right]$, ou encore :

$C_{1}=k_{1} k_{2} G R Z_{1} \rho /\left[P_{1}\left(Z_{1}+R\right)+P_{1} p_{1}-p_{1}^{2}\right]$.

$C_{1}$ est donc fonction de $R$ et de $p_{1}$. Pour que sa variation totale ne soit pas supérieure au rapport de 1 à 4 (cf. (4)), nous imposons une variation maximale de 1 à 2 en fonction de $p_{1}$ et également de 1 à 2 en fonction de $V_{\mathrm{D}}$.

2.1.1. Condition imposée d̀ $R$, avec $p_{1}$ variable.

$C_{1}$ est maximum quand $p_{1}=0$ ou $p_{1}=P_{1}$; on a alors :

$$
C_{1 \max }=k_{1} k_{2} G \rho R Z_{1} / P_{1}\left(Z_{1}+R\right) .
$$

$C_{1}$ est minimal quand $p_{1}=P_{1} / 2$; on a alors :

$$
C_{1 \min }=k_{1} k_{2} G \rho R Z_{1} I\left[P_{1}\left(Z_{1}+R\right)+\frac{P_{1}^{2}}{4}\right] \text {. }
$$

Nous réécrivons donc la première condition :

$$
C_{1 \text { max }} / C_{1 \min }=\frac{Z_{1} / P_{1}\left(Z_{1}+R\right)}{Z_{1} /\left[P_{1}\left(Z_{1}+R\right)+P_{1}^{2} / 4\right]}<2
$$

ce qui donne, après simplification :

$$
R>\left(P_{1} / 4\right)-Z_{1}
$$

2.1.2. $\mathrm{Si}$, maintenant, on envisage $V_{\mathrm{D}}$ variable, on écrit :

$$
C_{1 \max }=\left(k_{1} k_{2} \rho G Z_{1} / P_{1}\right) \cdot\left[R /\left(Z_{1}+R\right)\right]
$$

on voit que pour que $C_{1 \min }>C_{1 \max } / 2$, il faut et il suffit que :

$$
R>Z_{1} \text {. }
$$

On choisit l'une ou l'autre des inégalités (6) ou (7), suivant les valeurs de $P_{1}$ et $Z_{1}$. La condition (4) est alors respectée, quel que soitt $V_{\mathrm{N}}$ et quel que soit $V_{\mathrm{D}}$, si l'amortissement critique $C_{0}$ est obtenu pour $V_{\mathbb{N}} / V_{\mathrm{D}}=0,5$.

3. Réalisation pratique. - Nous utilisons des appareils anciens dont les caractéristiques sont les suivantes : $P_{\mathbf{1}}=1200 \Omega$; l'amplificateur différentil est shunté pour que $Z_{1}=400 \Omega$.

Nous avons fixé la plus petite valeur $\operatorname{de}^{-} R$ à $1000 \Omega$, donc bien supérieure à $Z_{1}$ (cf. (7)). Comme nous voulions une précision de $1 \%$, nous avons adapté un rhéostat de $100000 \Omega$.

$C_{1}$ est maximal pour $R$ maximal et $p_{1}$ maximal, d'où :

$C_{1 \max }=\left(k_{1} k_{2} \rho G Z_{1} / P_{1}\right) \cdot[100000 /(400+100000)]$.

$C_{1}$ est minimal pour $R$ minimal et $p_{1}=P_{1} / 2$, d'où :

$C_{1 \min }=\left(k_{1} k_{2} \rho G Z_{1} / P_{1}\right)$

$$
\cdot\left[1000 /\left(400+1000+\frac{1200}{4}\right)\right]
$$

$C_{1}$ varie donc dans un rapport de 0,588 à 0,996 .

Manuscrit reçu le 21 janvier 1966. 